\title{
A novel method to stimulate lymphatic clearance of beta-amy- loid from mouse brain using non-invasive music-induced open- ing of the blood-brain barrier with EEG markers
}

\author{
Oxana Semyachkina-Glushkovskaya ${ }^{1,2 *}$, Alexander Khorovodov' ${ }^{2}$ Ivan Fedosov², Alexey Pavlov' ${ }^{2}$ Alexander Shiro- \\ kov $^{2,3}$, Ali Esmat Sharif ${ }^{2}$, Alexander Dubrovsky' ${ }^{2}$ Inna Blokhina ${ }^{2}$, Andrey Terskov ${ }^{2}$, Nikita Navolokin ${ }^{2,4,5}$, Arina Evsu- \\ kova $^{2}$, Georgy Karandin ${ }^{2}$, Dariya Elovenko ${ }^{2}$, Maria Tzoy², Vasily Ageev ${ }^{2}$, Ilana Agranovich ${ }^{2}$, Valeria Telnova ${ }^{2}$, Anna \\ Tsven $^{2}$, Ali Esmat ${ }^{2}$, Elena Saranceva², Tatyana Iskra², Jürgen Kurths ${ }^{2,6}$ \\ 1 Humboldt University, Newtonstrasse 15, Berlin, Germany, 12489 \\ 2 Saratov State University, Astrakhanskaya 82, Saratov, Russia, 410012 \\ 3 Institute of Biochemistry and Physiology of Plants and Microorganisms, Russian Academy of Sciences, \\ Prospekt Entuziastov 13, Saratov, Russia, 410049 \\ 4 Saratov Medical State University, Kazachaya 112, Saratov, Russia, 410012 \\ 5 Saratov City Clinical Hospital No. 1 named after Yu.Ya. Gordeev, Kholzunova A.I. 19, Saratov, Russia, \\ 410017 \\ 6 Potsdam Institute for Climate Impact Research, Telegrafenberg A31, Potsdam, Germany, 14473 \\ * Correspondence: glushkovskaya@mail.ru; Tel.: +7 8452519220
}

\begin{abstract}
The lymphatic system of the brain meninges and head plays a crucial role in the clearance of amyloid- $\beta$ protein $(A \beta)$, a peptide thought to be pathogenic in Alzheimer's disease (AD), from the brain. The development of methods to modulate lymphatic clearance of $A \beta$ from the brain coild be a revolutionary step in the therapy of AD. The opening of the blood-brain barrier (OBBB) by focused ultrasound is considered as a possible tool for stimulation of clearance of $A \beta$ from the brain of humans and animals. Here, we propose an alternative method of non-invasive music-induced OBBB that is accompanied by the activation of clearance of fluorescent $A \beta(F \alpha \beta)$ from the mouse brain. Using confocal imaging, fluorescence microscopy and magnetic resonance tomography, we clearly demonstrate that OBBB by music stimulates the movement of $F \alpha \beta$ and Omniscan in the cerebrospinal fluid and lymphatic clearance of $\mathrm{F} \alpha \beta$ from the brain. We propose the extended detrended fluctuation analysis (EDFA) as a promising method for the identification of OBBB markers in the electroencephalographic (EEG) patterns. These pilot results suggest that music-induced OBBB and the EDFA analysis of EEG can be a non-invasive, low cost, labelling free, clinical perspective and completely new approach for the treatment and monitoring of $\mathrm{AD}$.
\end{abstract}

Keywords: music, blood-brain barrier, lymphatic system, amyloid- $\beta$ protein, detrended fluctuation analysis, electroencephalographic patterns.

\section{Introduction}

Cerebrospinal fluid (CSF) plays an important role in the movement of nutrients and waste products via the perivascular spaces (PVSs) [1-4]. The (re) discovery of the meningeal lymphatic vessels (MLVs) has led to a reassessment of the pathways for the clearance of waste from the central nervous system (CNS) [5,6]. Nowadays, the role of MLVs in brain functions, specifically in the context of ageing and Alzheimer's disease (AD), has been actively studied [7]. Alzheimer's disease is the most common form of dementia and its prevalence increases with age $[8,9]$. The extracellular deposition of amyloid- $\beta(A \beta)$ aggregates, the main constituent of senile plaques, is considered to be a pathological hallmark of $\mathrm{AD}$ that contributes to neuronal dysfunction and behavioral changes $[9,10]$. It is interesting to note that $A \beta$ was initially isolated from homogenates of meningeal tissue from patients with $\mathrm{AD}[11]$. Iliff et al. also demonstrated that fluorescent-tagged $\mathrm{A} \beta(\mathrm{F} \alpha \beta)$ 
is transported along PVSs and the glymphatic pathway, which can may remove F $\alpha \beta$ from the CNS [1].

The development of methods to modulate glymphatic/lymphatic clearance of $A \beta$ from the brain could become a revolutionary step in the therapy of AD. Currently, there are no pharmacological drugs that provide an effective therapy of $\mathrm{AD}$ and limit the development of cognitive impairment [12]. Note that pharmaceutical companies such as Biogen, Johnson \& Johnson, Pfizer announced the cancellation of funding for the synthesis of antibodies for the treatment of AD due to the failure of clinical trials [13]. Obviously, in the next couple of decades, the main strategies for a treatment of $\mathrm{AD}$ will be non-invasive methods of stimulation of clearance of the toxic $A \beta$ from the brain tissues.

The opening of the blood-brain barrier (OBBB) using focused ultrasound (FUS) is considered as very promising tool for the stimulation of clearance of $A \beta$ from the brain of humans and animals [14-19]. In patients with AD, OBBB by FUS causes a decrease in the level of amyloid deposition that was confirmed by [18F]-florbetaben positron emission tomography [14]. In transgenic mouse models of AD, FUS was used to deliver antibodies against $A \beta$ and tau, with significant reductions in pathology and a positive impact on memory performance $[15,16]$. Further studies in mouse models of amyloidosis demonstrated that even without exogenous antibody administration, BBB disruption by FUS reduced plaque burden, triggered neuronal plasticity, and prevented spatial memory deficits [17-19]. However, FUS remains largely experimental owing to a range of limitations: (1) FUS is not effective without gas microbubbles, which have low circulation times because they get taken up by immune system cells or by the liver or spleen [20]; (2) microbubbles burst at low ultrasound powers, which are associated with the activation of sterile inflammation ]21] and the appearance of apoptotic cells [22].

In our recent experimental study, we discovered the phenomenon of short lasting OBBB in healthy rodents induced by loud rock music [23] or sound [24]. Despite the fact that music opens the BBB in a general location-unspecified manner, loud music has a high potential for clinical applications as an easily used, non-invasive, low cost, labelling free, perspective and completely new approach for the treatment of $\mathrm{AD}$, which is associated with injuries of many brain regions and diffusive progression without specificity of the brain areas.

Currently, it has been discussed that the electroencephalographic (EEG) dynamics can be a potential biomarker of BBB integrity [25]. In our preliminary studies, we were demonstrated the successful application of nonlinear analysis of EEG patterns in the evaluation of OBBB [26-31]. We have found strong evidence that the EEG dynamics can be an important informative platform of drainage and clearing functions of the brain. So, OBBBmediated clearance of macromolecules from the brain $[23,24,32]$ is associated with a low frequency EEG brain activity in both humans and animals [26-31,33].

In this study on mice, we study the effects of music-induced OBBB on the drainage and clearing function of the brain and analyze the EEG structure associated with these processes. Using confocal imaging and magnetic resonance tomography (MRI), we clearly demonstrate that OBBB by music stimulates the movement of F $\alpha \beta$ and Omniscan in CSF and the lymphatic clearance of $F \alpha \beta$ from the brain. We propose the extended detrended fluctuation analysis (EDFA) as a promising method for the identification of OBBB markers in the EEG patterns.

\section{Materials and Methods}

\subsection{Subjects and groups}

The experiments were conducted on male mongrel mice $(20-25 \mathrm{mg})$. All procedures were performed in accordance with the Guide for the Care and Use of Laboratory Animals. The experimental protocols were approved by the Local Bioethics Commission of the Saratov State University (Protocol No. 7). The experiments were performed in the groups: (1 and 2) the control, including mice with intact BBB (IBBB) and intravenous injection of Evans Blue dye (EBD, $n=10)$ and $F \alpha \beta(n=10)$, respectively; ( 3 and 4$)$ mice with 
music-induced OBBB for EBD, n=10 and for FITC-dextran $70 \mathrm{kDa}, \mathrm{n}=10$, respectively. The groups 1-4 were used for the study of EEG structure using EDFA; (5) the control, including mice with IBBB and intraventricular injection of $\mathrm{F} \alpha \beta, \mathrm{n}=10$; (6) mice with $\mathrm{OBBB}$ and intraventricular injection of $\mathrm{F} \alpha \beta, \mathrm{n}=10$; (7) the control, including mice with IBBB and injection of Omniscan in the cortex, $\mathrm{n}=7$; (8) mice with OBBB and injection of Omniscan, $\mathrm{n}=7$.

\subsection{Model of music-induced $O B B B$}

The detailed description of our method of music-induced OBBB is present in Ref. [23]. To produce music (100 dB and 11-10,000 Hz, Scorpions "Still loving you") we used loudspeaker (ranging of sound intensity - 0-130 dB, frequencies - 63-15000 Hz; $100 \mathrm{~V}$, Yerasov Music Corporation, Saint Petersburg, Russia). The repetitive music exposure was performed using the following sequence: $1 \mathrm{~min}$ - music on, then $1 \mathrm{~min}$ - music off during $2 \mathrm{~h}$. The sound level was measured directly in a cage of animals using the sound level meter (Megeon 92130, Russia).

\subsection{Spectrofluorometric assay of Evans Blue dye (EBD) extravasation}

EBD (Sigma Chemical Co., St. Louis, Missouri, $2 \mathrm{mg} 25 \mathrm{~g}$ mouse, 1\% solution in physiological $0.9 \%$ saline) was injected into the femoral vein and circulated in the blood for $30 \mathrm{~min}$. Then, the rats were decapitated, and their brains were quickly collected. At the end of the circulation time, mice were decapitated, their brains and blood were quickly collected and placed on ice (no anti-coagulation was used during blood collection). Prior brain removal, the brain was perfused with saline to wash out remaining dye in the cerebral vessels. The level of EBD in the brain was evaluated in accordance with the recommended protocol [34].

\subsection{Monitoring the distribution of $F \alpha \beta$ and Omniscan in the mouse brain}

The Hamilton syringe ( $25 \mu \mathrm{l}$, Hamilton Co, Reno, Nevada, US) with the needle (25 Gauge) was mounted onto the injection pump using the stereotactic manipulator (Stoelting, New York, US). A small cranial burr hole was drilled through the skull using a variable speed dental drill (with a $1 \mathrm{~mm}$ drill bit). $5 \mu \mathrm{l}$ of beta-amyloid (1-42), HiLyte ${ }^{\mathrm{TM}}$ Fluor 488-labeled (AnaSpec Inc., Fremont, California, USA) at a rate of $0.1 \mu \mathrm{l} / \mathrm{min}$ was injected into the right lateral ventricle (AP-1.0 mm; ML-1.4 mm; DV-3.5 mm). The needle was left in the ventricle for $10 \mathrm{~min}$ and then removed at a rate of $1 \mathrm{~mm} / \mathrm{min}$ to prevent the reflux of $F \alpha \beta$. The burr hole and scalp incision were closed with bone wax (Ethicon, Somerville, NJ) and with cyanoacrylate glue (Henkel Consumer Adhesive Inc. Scottsdale, Arizona), respectively.

The real time and ex vivo optical study of the distribution of $F \alpha \beta$ was performed immediately (for in vivo) and $1 \mathrm{~h}$ (for ex vivo) after intraventricular injection of $\mathrm{F} \alpha \beta$ in mice with IBBB and OBBB. The $1 \mathrm{~h}$ was selected as the time of OBBB, which was established in our previous studies $[23,24]$. The imaging was performed using homemade optical instrument based on the monochrome camera ac A2040-90 um (Basler AG, Germany) and 50 mm 2,8 C- mount CCTV objective lens (Tamron, Japan) The lens was attaché o the camera with $15 \mathrm{~mm}$ extension tube to ensure macro imaging with 23.3 to $31.8 \mathrm{~mm}$ field of view depending of the lens focusing ring adjustment. The lens was mounted on the vertical manual translation stage (Standa, Lituania) above a Petri dish where samples were submerged in a buffer solution. The top surface of each sample was covered with $25 \times 50 \times 0.17$ mm cover glass. The slider with filter sets $(49019,49002$, Chroma Technology, USA) was placed just below the objective lens. Each filter set was illuminated with homemade condensers with 1W LEDs (635 nm for 49019; and $460 \mathrm{~nm}$ for 49002) to ensure uniform illumination over the camera field of view. Led illuminators were synchronized with camera "fire" output.

The camera resolution was $2048 \times 2048$ pixels at 12 bit grayscale. Images were acquired in a dark room at constant exposure time of $200 \mathrm{~ms}$ and other settings kept unchanged for all samples. An image acquisition and processing were performed with cus- 
tom software developed using the N I Vision and LabVIEW software (National Instruments, USA) and the FIJI open source image processing package [35]. Image processing procedures were identical for each pair of images (control and laser treated sample) for each channel to ensure an accurate comparison of the fluorescence intensity.

The analysis of $F \alpha \beta$ in the brain slices was carried out on a fluorescence microscopic system described above. For a quantitative analysis of the intensity signal from $F \alpha \beta$, ImageJ was used for image data processing and analysis. The intensity of fluorescence for each slide was integrated over a rectangular region of interest bounding brain slice. The integral value was divided by slice area. The areas of brain slices were calculated using the plugin "Analyze Particles" in the "Analyze" tab, which calculates the total area of F $\alpha \beta$ fluorescence intensity tissue elements - the indicator "Total Area". In all cases, 10 regions of interest were analyzed.

The distribution of the MRI contrast agent Omniscan ${ }^{\circledR}$ (GE Health Care, US, 0.1 $\mathrm{mmol} / \mathrm{kg}$ ) in the mouse brain after its injection into the cortex (AP - $1.06 \mathrm{~mm} ; \mathrm{DV}-1.0$ $\mathrm{mm}$; ML $-1.5 \mathrm{~mm}$ ) was studied during 2 hrs using an ultra-high-field tomography BioSpec 117/16 USR (11 T, Bruker, Germany). Mice were anaesthetised with $\%$ isoflurane at 1L/min N2O/O2 - 70:30 (Isoflurane, Baxter Healthcare Corp., USA) using an anaesthesia machine (The Univentor 400 Anaesthesia Unit, Univentor, Malta). The temperature of the animals was maintained using a water circuit in a tomographic bed-table with a surface temperature of $30^{\circ} \mathrm{C}$. A pneumatic breathing sensor (SA Instruments, Stony Brook, N.Y., USA) was placed under the lower torso, which made it possible to control the depth of anaesthesia.

Information on the distribution of contrast in the mouse brain was investigated using T1-weighted images obtained using the RARE method (Rapid Acquisition with Relaxation Enhancement). The pulse sequence parameters of the method (TE $=10 \mathrm{~ms}, \mathrm{TR}=400$ $\mathrm{ms}$ ), the image parameters (size $1.8 \times 1.8 \mathrm{~cm}$; matrix $256 \times 256$ points; slice thickness 0.5 $\mathrm{mm}$; voxel dimensions $75 \mu \mathrm{m} \times 75 \mu \mathrm{m} \times 0.5 \mathrm{~mm}$; the distance between the slices is 0.5 $\mathrm{mm}$; the number of slices is 9; the orientation of the slices is coronal), and the total scanning time was $7 \mathrm{~min}$.

The accumulation of MRI contrast was expressed as the ratio of the level of the MRI signal in the studied structures to the level of the MRI signal in the reference, which was a phosphate-buffered microtube $(0.5 \mathrm{ml})$ placed along the head of the mouse. The MRI scans were processed using the ImageJ software.

\subsection{EEG and EMG recording}

A two-channel cortical EEG/one channel electromyogram (EMG) (Pinnacle Technology, Taiwan) were recorded. The mice were implanted two silver electrodes (tip diameter 2-3 $\mu \mathrm{m}$ ) located at a depth of $150 \mu \mathrm{m}$ in coordinates (L: $2.5 \mathrm{~mm}$ and D: $2 \mathrm{~mm}$ ) from Bregma on either side of the midline under inhalation anesthesia with $2 \%$ isoflurane at $1 \mathrm{~L} / \mathrm{min}$ $\mathrm{N}_{2} \mathrm{O} / \mathrm{O}_{2}-70: 30$. The head plate was mounted and small burr holes were drilled. Afterward, EEG wire leads were inserted into the burr holes on one side of the midline between the skull and the underlying dura. EEG leads were secured with dental acrylic. An EMG lead was inserted in the neck muscle. Ibuprofen $(15 \mathrm{mg} / \mathrm{kg})$ for the relief of postoperative pain was provided in their water supply for two to three days prior to surgery and for three or more days post-surgery. The mice were allowed 10 days to recover from surgery prior to the beginning of the experiment.

\subsection{Extended detrended fluctuation analysis of EEG structure}

There are several reasons that complicate the quantification of long-range power-law correlations in physiological data sets, namely, the nonstationarity and the fast rate of the decay of the correlation function. The latter produces values close to zero, where computational errors can strongly affect the results. Due to problems, the analysis of very slow physiological processes in terms of the correlation function is ineffective, and alternative approaches are necessary. One such approach is the detrended fluctuation 
analysis (DFA), which was proposed by Peng et al $[36,37]$ to improve the correlation analysis of natural processes. This method can be treated as a version of the root mean square study of a signal profile.

The data set $x(i), i=1, \ldots, N$ is analyzed according to the following algorithm:

1) The creation of a random walk $y(k)$ associated with the original dataset

$$
y(k)=\sum_{i=1}^{k}[x(i)-\langle x\rangle]
$$

where $\langle x\rangle$ is the mean value of $x(i)$.

2) Segmentation of $y(k)$ into fragments of size $n$ and estimation of the local trend $y_{n}(k)$ in every segment using linear fitting.

3) Computation of the root mean-square fluctuation

$$
F(n)=\sqrt{\frac{1}{N} \sum_{k=1}^{N}\left[y(k)-y_{n}(k)\right]^{2} \sim n^{\alpha} .}
$$

Such power-law behavior (eq. 2) is typical for many natural systems. Consideration of this dependence on a double logarithmic plot provides an ability to estimate the scaling exponent $\alpha$, whose value is nearly constant for homogeneous datasets but can vary with $n$ for inhomogeneous processes.

Using $\alpha$, the correlation analysis of nonstationary processes can be provided, where $\alpha<0.5$ quantifies the alternation of large and small values (anti-correlations), $\alpha=0.5$ indicates the absence of correlations, and $\alpha>0.5$ marks different types of positive correlations, which are related to power-law behavior $(\alpha<1)$, and a behavior that may differ from power-law statistics $(\alpha>1)$.

The above standard DFA algorithm does not account for possible changes in nonstationary behavior across the entire dataset, which are clearly pronounced for transients, intermittency, or abrupt changes in energy as a typical in EEG data. To adapt the DFA tool to the case of variable nonstationary properties, we have proposed an extended DFA (EDFA) [38]. Its main idea is to account for local distinctions in the RMS deviation (2) between different parts of the dataset, i.e. to consider the inhomogeneous structure of the signals. For this purpose, a new measure is introduced

$$
d F(n)=\max \left[F_{l o c}(n)\right]-\min \left[F_{l o c}(n)\right]
$$

It characterizes the impact of nonstationarity and determines the difference between the extreme values of local RMS deviations. If nonstationarity does not show significant changes between different parts of the data, measure (3) does not take large values. However, for clearly inhomogeneous datasets, $d F(n)$ can show strong distinctions. Typically, the dependence (3) shows a power-law behavior, although the related scaling exponent is different 


$$
d F(n) \sim n^{\beta}
$$

The given approach has been successfully applied in several our studies [28, 31].

\subsection{Immunohistochemical assay}

Mice from the groups of 2 and 4 were euthanized with an intraperitoneal injection of a lethal dose of ketamine and xylazine and intracardially perfused with $0.1 \mathrm{M}$ of phosphate-buffered saline (PBS) for $5 \mathrm{~min}$. Afterward, the brains, the meninges and the deep cervical lymph nodes (dcLNs) were removed and fixed in $4 \%$ buffered paraformaldehyde for one day and in $20 \%$ sucrose for another day. The meninges were removed and fixed as described in [39]. The signal intensity from the examined proteins was evaluated on free-floating sections using the standard method of simultaneously combined staining (Abcam Protocol). Brain slices $(50 \mu \mathrm{m})$ were blocked in $150 \mu \mathrm{l}$ 10\% BSA/0.2\% Triton X100/PBS for $2 \mathrm{~h}$, then incubated overnight at $4 \mathrm{C}$ and $2 \mathrm{~h}$ at room temperature with lymphatic vessel endothelial hyaluronan receptor 1 (Lyve-1) (1:500; Invitrogen, Molecular Probes, Eugene, Oregon, USA), glial fibrillary acidic protein (GFAP) (1:500; Abcam, Cambridge, UK); prospero homeobox protein 1 Prox-1 (1:500; Invitrogen, Molecular Probes, Eugene, Oregon, USA), endothelial cell adhesion molecule 1 CD31 (1:500; Invitrogen, Molecular Probes, Eugene, Oregon, USA). After several rinses in PBS, the slides were incubated for $3 \mathrm{~h}$ at room temperature with fluorescent- labeled secondary antibodies on $1 \%$ BSA/0.2\% Triton X-100 /PBS (1:500; Goat A/Rb, Alexa 405 and 647 Abcam, UK). Confocal microscopy of the cerebral cortex, the meninges and dclNs was performed using a confocal microscope with water immersion Olympus FV10i-W (Olympus, Japan). In all cases, 10 regions of interest were analyzed.

\subsection{Statistical analysis}

The experimental data are presented as mean \pm standard error of the mean (SEM). Differences from the initial level in the same group were evaluated by the Wilcoxon test. Intergroup differences were evaluated using the Mann-Whitney test and ANOVA-2 (post hoc analysis with Duncan's rank test). The significance levels were set at $p<0.05-0.001$ for all analyses.

\section{Results}

\subsection{Music-induced OBBB}

In the first step, we performed a qualitative and quantitative analysis of the BBB disruption using the intravenous injection of EBD (961 Da). The EBD is a commonly used tracer for the estimation of the BBB disruption because it is a non-toxic and not metabolically active dye, which rapidly binds to serum albumines creating the high molecular weight Evans Blue-albumin complex (EBAC, $68.5 \mathrm{kDa}$ ). The IBBB is impermeable to EBAC and its extravasation into the brain parenchyma indicates the BBB disruption $[23,24,34$, 40-42].

We used spectrofluorimetry for the EBAC quantitation $1 \mathrm{~h}$ and $4 \mathrm{~h}$ after music impact. This time was chosen based on the previous data on mice, in which we showed that the BBB opens $1 \mathrm{~h}$ after music effects and the BBB closes $4 \mathrm{~h}$ after music listening [23]. Our results clearly show that music caused OBBB for EBAC in mice $1 \mathrm{~h}$ after music action that was associated by an accumulation of EBAC in the brain parenchyma vs. intact mice $(2.84 \pm 0.14 \mu \mathrm{g} / \mathrm{g}$ tissue vs. $0.12 \pm 0.05 \mu \mathrm{g} / \mathrm{g}$ tissue, $\mathrm{p}<0.001, \mathrm{n}=10$ in each group). There were no any changes in the EBAC levels in the brain parenchyma between the control mice and mice, whose brain were taken $4 \mathrm{~h}$ after listening of music $(0.14 \pm 0.10 \mu \mathrm{g} / \mathrm{g}$ tissue vs. $0.11 \pm 0.01 \mu \mathrm{g} / \mathrm{g}$ tissue, $\mathrm{n}=10$ in each group). Figure $1 \mathrm{a}$ and $\mathrm{b}$ illustrates an qualitative analysis of the BBB permeability to FITC-dextran $70 \mathrm{k}$ Da using confocal microscopy. This 
method also revealed no BBB disruption before music impact (Fig. 1a) and distinct distribution of FITC-dextran (Fig. 1b) among the cerebral vessels and astrocytes in mice 1h after music effects. These data are consistent with our previous results demonstrating musicinduced OBBB to the high molecular weight substances in 11 brain regions, including frontal, parietal, occipital, temporal, cingulate cortex, hypothalamus, thalamus, hippocampus, inferior and superior colliculi, cerebellum, pons, and medulla [23]. Thus, these results confirm the highly reproducibility of non-invasive method of reversible OBBB by music in mice.

\subsection{Music-induced $O B B B$ stimulates clearance of Faß from the mouse brain}

Next, we investigated how OBBB changes the movement of Fa $\beta$ in CSF. Figures $1 \mathrm{c}$ and $d$ illustrate the real time fluorescence microscopy of fast Fa $\beta$ spreading from the ventricle to the subarachnoid space in mice with IBBB and OBBB. The intensity of the fluorescent signal was monitored immediately after $\mathrm{Fa} \beta$ injection into the right lateral ventricle of mice from both groups. Our results show that mice with OBBB vs. mice with IBBB demonstrated a higher fluorescent signal from $\mathrm{Fa} \beta$ on the brain surface. Ma et al. showed a characterization of time-depended CSF flow routes within the mouse cranium [43]. They clearly demonstrated that after intravenricular injection, the tracer distributes from the dorsal to ventral aspects of the brain. Based on these facts, in the next step, we analyzed a delayed distribution of $\mathrm{Fa} \beta \mathrm{h}$ after its injection in the ventricle using ex vivo fluorescence microscopy. Figure 1 e-h illustrate that mice with OBBB vs. mice with IBBB demonstrated faster distribution of $\mathrm{Fa} \beta$ from the dorsal to lateral aspects of the brain. Indeed, the intensity of the fluorescent signal from $\mathrm{Fa} \beta$ was 2.3-fold lesser on the dorsal aspect of the brain $(0.11 \pm 0.03$ a.u. vs. $0.25 \pm 0.03$ a.u., $\mathrm{p}<0.001)$ and 3.5 -fold higher on the ventral part of the brain $(0.42 \pm 0.01$ a.u. vs. $0.12 \pm 0.02$ a.u., $\mathrm{p}<0.001)$ in mice with $\mathrm{OBBB}$ vs. mice with IBBB (Figure $1 \mathrm{q}$ ). These data suggest that OBBB contributes to the distribution of Fa $\beta$ from the ventricle to the area of location of the basal MLVs playing an important role in the brain drainage $[5,6,43]$. Figures 1 i shows the perivascular pathway of Fa $\beta$ spreading. Notice that perivascular and paravascular routes are considered as the crucial pathways of fluid drainage and clearance of toxins from the brain $[1-4,44]$. We also observed lymphatic clearance of $\mathrm{Fa} \beta$ from the mouse brain that was faster in mice with OBBB than in animals with IBBB. Figure $1 \mathrm{j}$ illustrates the presence of $\mathrm{Fa} \beta$ in MLVs that we observed in both group. This fact suggests that MLVs are pathway of clearance of $\mathrm{Fa} \beta$ from the brain and confirms results of others $[7,11]$ (Figure $1 \mathrm{j}$ ). However, mice with OBBB demonstrated the a stronger intensity of signal from $\mathrm{Fa} \beta$ in the meninges than in mice with IBBB (Figures 1 $\mathrm{k}$ and 1 ). The dcLNs are the first anatomical station of CSF exit from the brain and the place of accumulation of molecules, which move from the CNS [5,7,23]. As shown in Figure 1 $\mathrm{m}, \mathrm{n}$ and $\mathrm{q}$ the accumulation of $\mathrm{Fa} \beta$ in dcLNs was higher in mice with OBBB vs. mice with IBBB. 


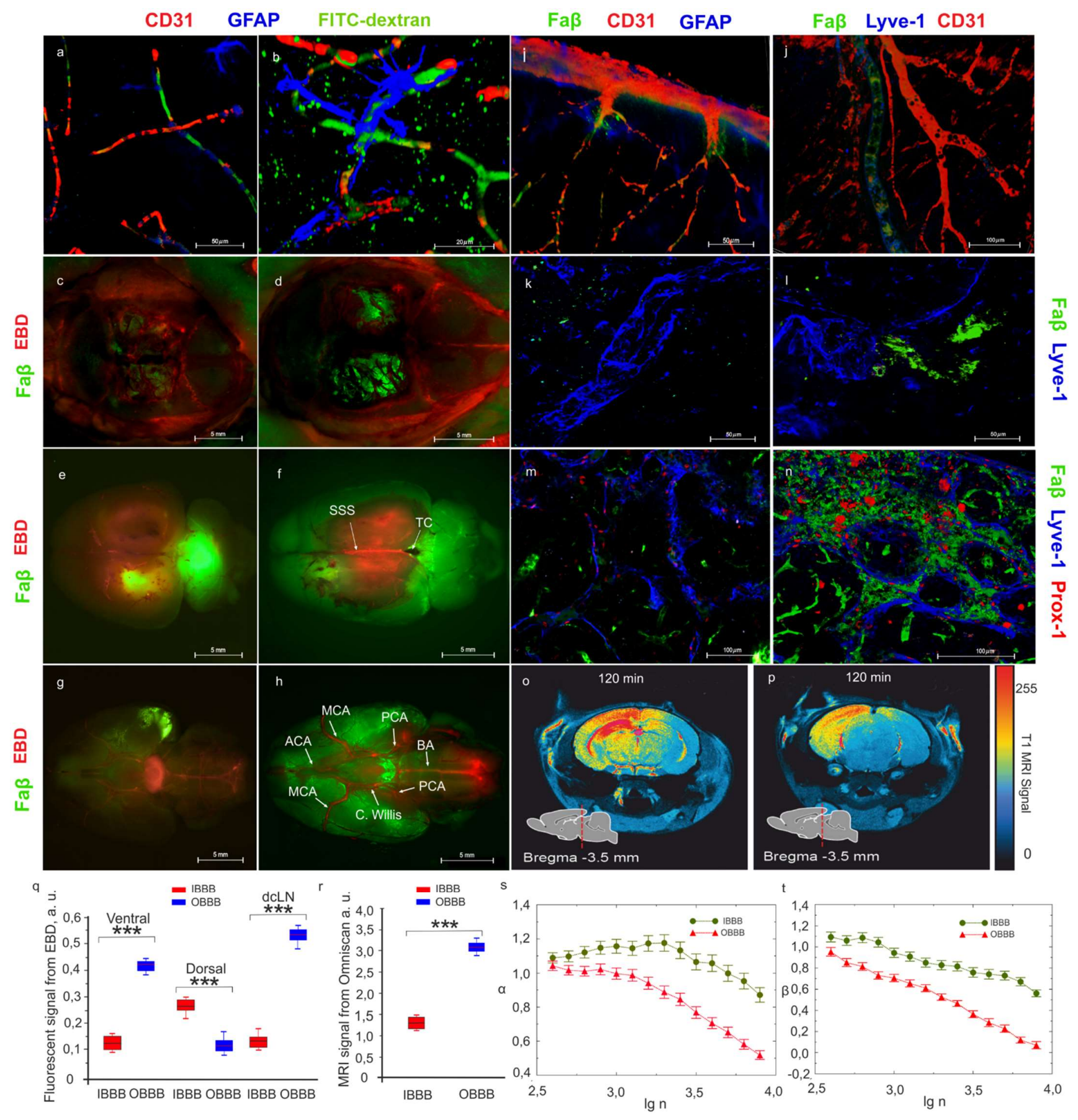

Figure 1. Music-induced OBBB affects the clearance of tracers from the mouse brain and the EEG structure: (a and b) Representative images of IBBB (a) and OBBB (b). In mice with IBBB, FITC-dextran is present in the cerebral vessels, while in mice with OBBB, the tracer is extravasated from the blood vessels and is distributed among the cerebral microvasculature and astrocytes; (c and d) Representative images of real time fluorescence imaging of fast distribution of Fa $\beta$ over the surface of the brain of mice with IBBB (c) and OBBB (d) immediately after injection of Fa $\beta$ in the right later ventricle; (e-h) Representative images of ex vivo fluorescence microscopy of delay distribution of $\mathrm{Fa} \beta$ in the lateral and dorsal parts of the brain of mice with IBBB (e and g) and OBBB ( $\mathrm{f}$ and $\mathrm{h}$ ) $1 \mathrm{~h}$ after injection of $\mathrm{Fa} \beta$ in the right later ventricle; (i and $\mathbf{j}$ ) Representative images of Fa $\beta$ distribution along the cerebral vessels in the pia matter (i) and in the meninges (j) in mice with OBBB; (k and $\mathbf{l})$ Representative images of Fa $\beta$ spreading in the meninges of mice with IBBB $(\mathrm{k})$ and OBBB $(\mathrm{l}) ;(\mathbf{m}$ and 
n) Representative images of presence of $\mathrm{Fa} \beta$ in dcLNs of mice with IBBB (m) and OBBB (n); (o and p) Representative images of MRI analysis of Omniscan distribution over the brain $2 \mathrm{~h}$ after its injection in the cortex of mice with IBBB (o) and OBBB (p); (q and r) Quantitative analysis of intensity of fluorescent signal from $\mathrm{Fa} \beta$ in a.u. on the ventral and dorsal aspects of the brain and in dcLNs in mice with IBBB and OBBB; (o and p) Quantitative analysis of MRI signal from Omniscan in a.u. in mice with IBBB (o) and OBBB (p); s and $\mathbf{t}$, Scaling exponents $\alpha$ and $\beta$ estimated as local slopes of the dependencies $F(n)$ and $d F(n)$ on the double logarithmic plot. Data are shown as mean values \pm SE (averaging over all available datasets). Local slopes were computed within a "window" of lgn equal to 0.6. $\mathrm{n}=10$ for $\mathrm{a}-\mathrm{n}, \mathrm{q}, \mathrm{s}$ and $\mathrm{t} ; \mathrm{n}=7$ for $\mathrm{o}, \mathrm{p}$ and $\mathrm{r}$, ${ }^{* *}-\mathrm{p}<0.001$, two-tailed Mann-Whitney U test for $\mathrm{q}$ and $\mathrm{r}$.

We next, analyzed the distribution of Omniscan after its injection in the cortex in mice with IBBB and OBBB using real time MRI imaging during 2 hours. Figure $1 \mathrm{o}, \mathrm{p}$ and $r$ demonstrate that the MRI signal from the contrast agent was 2.1-fold higher in mice with $\mathrm{OBBB}$ vs. mice with IBBB (3.20 \pm 0.03 a.u. vs. $1.47 \pm 0.05$ a.u., $\mathrm{p}<0.001)$.

Altogether, these results suggest that OBBB increases the movement of $\mathrm{Fa} \beta$ in CSF and lymphatic clearance of $\mathrm{Fa} \beta$ from the brain.

\subsection{OBBB-induced changes in the structure of EEG signals}

Here we used EDFA charactering the changes in the scaling exponents $\alpha$ and $\beta$ for the analysis of EEG dynamic associated with OBBB and activation of drainage and clearance of the brain. Typically, OBBB is accompanied by a decrease in the slopes of the $F(n)$ or $d F(n)$ dependencies (eq. 2 and 3), although the related scaling exponents can vary depending on the scale $[26,31,30]$. This circumstance can be explained by the complex (multiscale) structure of EEG signals that need a number of measures for a complete quantitative assessment of their features. The latter is fairly typical for multifractal processes, and many physiological datasets clearly demonstrate such complex behavior. In terms of numerical measures, this inhomogeneity means that the scaling exponents may differ depending on the timescale, and the local slopes of $F(n)$ and $d F(n)$ in the double logarithmic plot may not match. Our estimates show such distinctions for both scaling exponents. Figure 1s and $t$ illustrate the local slopes of $\lg F$ and $\lg d F$ depending on $\lg n$. These estimates are carried out in the "window" of $\lg n$ equal to 0.6 . They show that both local exponents, $\alpha$ and $\beta$, usually decrease for OBBB vs. IBBB. This decrease is well expressed in a wide range of $\lg n$ and, therefore, clear changes in the structure of EEG data are revealed. The most pronounced distinctions are associated with the range $\lg n \in[3.3 ; 4.0]$. For example, when considering $\lg n=3.5$, the measures reduce $(p<0.01)$ from $\alpha=1.07 \pm 0.05, \beta=0.76 \pm 0.04$ (IBBB) to $\alpha=0.78 \pm 0.04, \beta=0.36 \pm 0.03$ (OBBB).

Although differences between states are observed for both scaling exponents, the relative changes are stronger for the $\beta$-exponent. According to Figure $1 \mathrm{~s}$, the $\alpha$-exponent of the conventional EDFA takes values in the ranges [0.52; 1.04] for OBBB, and [0.87; 1.19] for IBBB. In the case of the $\beta$-exponent, the related ranges are $[0.07 ; 0.95]$ for OBBB, and $[0.56 ; 1.09]$ for IBBB. Note that this measure can show not only quantitative, but also qualitative changes, consisting in a change in the sign of $\beta$, and some animals clearly demonstrate a transition from a positive slope of $\lg d F$ vs. $\lg n$ associated with IBBB, to a negative slope related to OBBB. These results allow us to conclude that (i) OBBB can be identified based on the EEG data by analyzing long-range power-law correlations, and (ii) the EEG markers of OBBB are clearly provided using the modified version of DFA, namely the EDFA. Moreover, the use of the additional scaling exponent that characterizes the impact 
of nonstationarity can improve the separation between physiological states based on the conventional approach.

\section{Discussion}

In this experimental work, we clearly demonstrate an efficiency of a novel method of stimulation of clearance of $F \alpha \beta$ from mouse brain using non-invasive music-induced OBBB. The $A \beta$ is present in the brain fluids and is regarded as a metabolic waste product [45]. For the last three decades, the $A \beta$ hypothesis, which posits $A \beta$ deposition as a key initial step in the pathogenesis of $\mathrm{AD}$, has been the dominant theory driving treatment development [46]. While the role of $\mathrm{A} \beta$ burden in the development and progression of clinical symptoms remains unclear and is under active investigation, the $\mathrm{A} \beta$ plaque clearance has been a key target of numerous clinical trials. Results to date have largely been disappointing $[47,48]$, although one recent trial linked a significant reduction in $A \beta$ to the stabilization of cognitive decline at 1 year [49]. Therefore, strategies that prevent A $\beta$ accumulation in the brain could promote healthy brain aging and be useful in preventing AD.

We revealed here that music-induced OBBB stimulates endogenous drainage and clearing processes in the mouse brain. Indeed, using in vivo and ex vivo fluorescence microscopy, we observed that spreading of F $\alpha \beta$ in CSF was higher in mice with OBBB vs. IBBB. Using an MRI-analysis of the distribution of Omniscan in the mouse brain, we also found that the distribution of the tracer was higher in mice with OBBB vs. IBBB. Our results are consistent with human data suggesting that OBBB by FUS stimulates an effective clearance of soluble $A \beta$ from the brain of patients with $A D$ that is associated with a significant improving of their cognitive status [14]. The mouse models of amyloidosis also show that FUS reduced the level of $\mathrm{A} \beta$ in the brain, triggered neural plasticity, and prevented spatial memory deficit [17-19].

The mechanisms by which $A \beta$ is cleared from the brain are not fully understood, although there is evidence that MLVs play an important role in removing $A \beta$ from the brain of humans and animals [7,11]. We investigated removing of A $\beta$ from the mouse brain via the meninges into dcLNs, which are the first anatomical station of CSF exit and the place of accumulation of molecules excreted from the brain [5,23]. Our results revealed that the level of the fluorescent signal from $\mathrm{F} \alpha \beta$ in both the meninges and dcLNs was significantly higher in mice with OBBB compared with mice with IBBB. These data suggest that OBBB is associated with an activation of lymphatic clearance of $F \alpha \beta$ from the mouse brain. In our previous investigations, we showed that music-induced OBBB is accompanied by fast removing of fluorescent dextran $70 \mathrm{kDa}$ after its crossing of OBBB [23].

We hypothesis that OBBB contributes to changes in PVS and in the extracellular spaces due to a transport via OBBB soluble molecules and fluids (Figure 2). Similar scenario is observed during sleep, when the volume of PVSs increases in 60\% [50]. These changes are strongly associated with the activation of clearance of A $\beta$ from the brain [50]. Opposite, wakening dramatically reduces the volume of PVS that is accompanied by the accumulation of $A \beta$ in the brain [50,51]. We assume that OBBB-induced changes in drainage of the brain tissues contributes lymphatic clearance of $A \beta$ from the brain. Further studies of this hypothesis will shed light on the mechanisms by which $A \beta$ is cleared from the brain. 


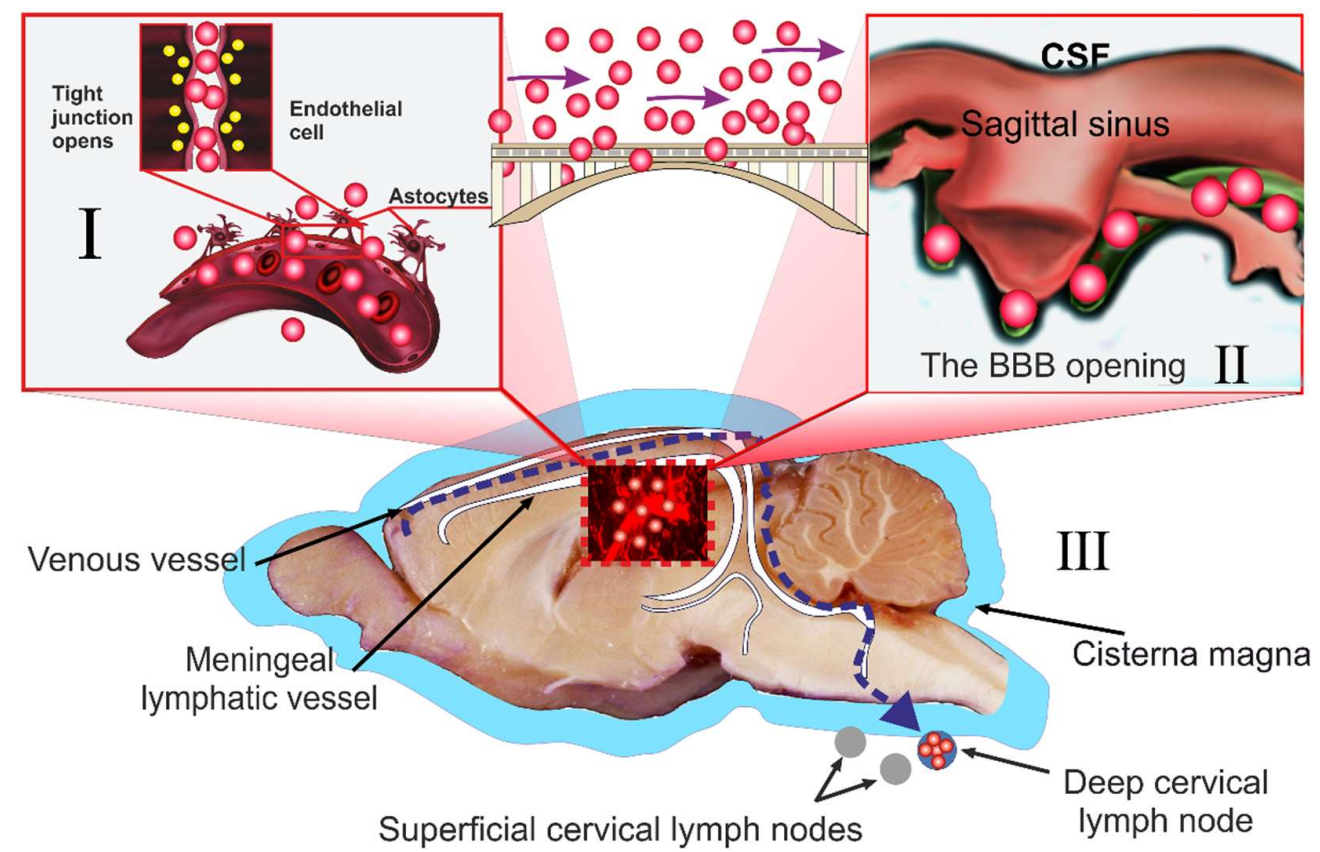

Figure 2. The illustration of hypothesis of OBBB-induced activation of drainage and clearance of the brain: I, The OBBB is accompanied by transport of soluble molecules and fluids from the blood vessels to the brain parenchyma; II, The MLVs are pathway of removing of molecules from the brain after its crossing OBBB; III, The dcLNs are the first anatomical station of lymphatic clearance of molecules crossing OBBB.

In our recent review, we discussed that the EEG dynamics can be a potential biomarker of BBB integrity [25]. Here we propose the EDFA analysis of EEG data and show that OBBB can be identified based on the EEG data using long-range power-law correlations. Our data concur with our previous results demonstrating a successful application of a nonlinear analysis of EEG patterns in the evaluation of OBBB [26-31]. These approaches open a new era in the development of non-invasive and bedside methods of express diagnosis of OBBB that is very important for monitoring the effectiveness of therapy and condition of patients with brain diseases associated with OBBB, such as AD, stroke, brain trauma, gliomas, neurodegenerative pathologies.

\section{Conclusions}

Here, we propose a novel method of non-invasive stimulation of clearance of $F \alpha \beta$ from the mouse brain using music-induced OBBB associated with significant changes in the EEG structure. Using fluorescence microscopy, confocal imaging and MRI, we clearly demonstrate that OBBB by music stimulates the movement of F $\alpha \beta$ and Omniscan in CSF and lymphatic clearance of $F \alpha \beta$ from the brain. We propose EDFA as a promising method for the identification of OBBB markers in EEG patterns. These pilot results suggest that music-induced OBBB and the EDFA analysis of EEG can be non-invasive, low cost, labelling free, clinical perspective and completely new approach for the treatment and monitoring of $\mathrm{AD}$.

Author Contributions: O.S-G. and J.K. initiated and supervised this work. A.Sh., A.T. and A.D. performed confocal analysis; I.F. performed analysis of distribution of $\mathrm{F} \alpha \beta$ in the brain using fluorescence microscopy; A.Kh made MRI scans; I.B., N.N., A.P., A.E., G.K., D.E., M.T., V.A., I.A., V.T., A.T., E.S., T.I. performed most of the experiments. E.S. prepared the figures. O.S-G. and J.K. reviewed all results and wrote the manuscript. J.K. participated in the discussions of the results and commented on the manuscript. All authors were also involved in the results discussion and manuscript reviewing and editing. 
Funding: S-G O., A.K, I.F., A.Sh., A.P., A.D., I.B., A.T., N.N., A.E., G.K., D.E., M.T., V.A., I.A., V.T., A.T., JK were supported by RF Governmental Grant № 075-15-2019-1885, Grant from RSF № 20-1500090; 21-75-10088, Grant from RFBR 19-515-55016; China a, 20-015-00308-a.

Institutional Review Board Statement: All procedures were performed in accordance with the "Guide for the Care and Use of Laboratory Animals". The experimental protocols were approved by the Bioethics Commission of the Saratov State University (Protocol No. 7).

Acknowledgments: We thank research center "Symbiosis" IBPPM RAS for their support with confocal microscopy and preparation of glioma cultures within Project No. GR 121031100266-3.

Conflicts of Interest: The authors declare that they have no competing interests.

\section{References}

1. Iliff, J. J.;Wang, M.; Liao, Y.; Plogg, B. A.; Peng, W.; Gundersen, G. A.; Benveniste, H.; Vates, G. E.; Deane, R.; Goldman, S. A.; Nagelhus, E. A; Nedergaard, M. A paravascular pathway facilitates CSF flow through the brain parenchyma and the clearance of interstitial solutes, including amyloid $\beta$. Sci. Transl. Med. 2012, 4, 147-147, DOI: 10.1126/scitranslmed.3003748.

2. Jessen, N. A. ; Munk, A. S. ; Lundgaard, I. ; Nedergaard, M. The glymphatic system: A beginner's guide. Neurochem. 2015, 40, 2583-2599, DOI 10.1007/s11064-015-1581-6.

3. Weller, R. O. .; Djuanda, E.; Yow, H. Y.; Carare, R. O. Lymphatic drainage of the brain and the pathophysiology of neurologi-cal disease. Acta Neuropathol. 2009, 117(1), 1, doi: 10.1007/s00401-008-0457-0.

4. Weller, R. O.; Galea, I. Carare, R. O.; Minagar, A. Pathophysiology of the lymphatic drainage of the central nervous system: Implications for pathogenesis and therapy of multiple sclerosis. Pathophysiology. 2010,17(4), 295-306, doi: 10.1016/j.pathophys.2009.10.007.

5. Louveau, A.; Smirnov, I.; Keyes, T. J.; Eccles, J.D.; Rouhani, S.J.; J. Peske, D.; Derecki, N.C.; Castle, D.; Mandell, J.W.; Lee, K.S.; Harris, T.H.; Kipnis, J.Structural and functional features of central nervous system lymphatic vessels. Nature.2015, 523, 337-341, doi.org/10.1038/nature14432.

6. Ahn, J.H.; Cho, H.; Kim, J.H; Kim, S.H.; Ham, J-S.; Park, I.; Suh, S.H.; Hong, S.P.; Song, J-H.; Hong, Y-K.; Jeong, Y.; Park, S-H.; Koh, G.Y. Meningeal lymphatic vessels at the skull base drain cerebrospinal fluid. Nature. 2019, 572, 62-66, doi.org/10.1038/s41586-019-1419-5.

7. Da Mesquita, S.; Louveau, A.; Vaccari, A.; Smirnov, i.; Cornelison, R.; Kingsmore, K.M.; Contarino, C.; OnengutGumuscu, S.; Farber,E.; Raper, D.; Viar, K.E.; Powell, R.D.; Baker, W.; Dabhi, N.; Bai, R.; Cao,R.; Hu, S.; Rich,S.S.; Munson,J.M.; Lopes, M.B.; Overall, C.C; Scott T. Acton, S.T.; Kipnis, J. Functional aspects of meningeal lymphatics in ageing and Alzheimer's dis-ease. Nature. 2018, 560, 185-191, doi.org/10.1038/s41586-018-0368-8.

8. Brookmeyer, R.; Abdalla, N.;Kawas, C. H.; Corrada, M. M. Forecasting the prevalence of preclinical and clinical Alz-heimer's disease in the United States. Alzheimers Dement. 2018, 14, 121-129, doi.org/10.1016/j.jalz.2017.10.009.

9. Erkkinen, M. G.; Kim, M. O.; Geschwind, M. D. Clinical neurology and epidemiology of the major neurodegenerative diseases. Cold Spring Harb. Perspect. Biol. 2018,10, a033118, doi: 10.1101/cshperspect.a033118.

10. Benilova, I.; Karran, E.; De Strooper, B.; The toxic A $\beta$ oligomer and Alzheimer's disease: an emperor in need of clothes. Nat. Neurosci. 2012, 15, 349-357, doi:10.1038/nn.3028.

11. Joachim, C. L.; Dufy, L. K.; Morris, J. H.; Selkoe, D. J. Protein chemical and immunocytochemical studies of meningovas-cular $\beta$-amyloid protein in Alzheimer's disease and normal aging. Brain Res. 1988,474, $100-111$.

12. Dunkel, P; Chai,C.L.; Sperlágh, B.; Huleatt, P.B.; Mátyus, P. Clinical utility of neuroprotective agents in neurodegen-erative diseases: current status of drug development for Alzheimer's, Parkinson's and Huntington's diseases, and amyotrophic lateral sclerosis. Expert Opin. Investig. Drugs. 2012, 21(9), 1267-1308, doi.org/10.1517/13543784.2012.703178 . 
13. Biogen/Eisai Halt Phase 3 Aducanumab Trials. https://www.alzforum.org/news/research-news/biogeneisaihaltphase-3-aducanumab-trial

14. Lipsman, N.; Meng, Y.; Bethune, A.; Huang, Y.; Lam, B.; Masellis, M.; Herrmann, N.; Heyn, C.; Aubert, I.; Boutet, A.; Smith, G.S.; Hynynen, K.; Black, S.E.. Blood-brain barrier opening in Alzheimer's disease using MR-guided focused ultrasound. Nat. Com-mun. 2018, 9, 2336, doi.org/10.1038/s41467-018-04529-6 .

15. Nisbet, R. M.; Van der Jeugd, A.; Leinenga, G.; Evans, H. T.; Janowicz, P. W.; Götz, J. Combined effects of scanning ultrasound and a tau-specific single chain antibody in a tau transgenic mouse model. Brain. 2017, 140, 1220-1230, doi.org/10.1093/brain/awx052.

16. Jordão, J.F.; Ayala-Grosso, C.A.; Markham, K.; Huang, Y.; Chopra, R.; McLaurin, J.; Hynynen, K.; Aubert I. Antibodies targeted to the brain with image-guided focused ultrasound reduces amyloid-beta plaque load in the TgCRND8 mouse model of Alzheimer's disease. PLoS ONE. 2010, 5, e10549, doi.org/10.1371/journal.pone.0010549 .

17. Jordão, J. F.; Thévenot, E.; Markham-Coultes, K.; Scarcelli, T.; Weng, Y. Q.; Xhima, K.; O'Reilly, M.; Huang, Y.; McLaurin, J.; Hynynen, K.; Aubert, I. Amyloid- $\beta$ plaque reduction, endogenous antibody delivery and glial activation by brain-targeted, tran-scranial focused ultrasound. Exp. Neurol. 2013,248, 16-29,doi.org/10.1016/j.expneurol.2013.05.008 .

18. Leinenga, G.; Götz, J. Scanning ultrasound removes amyloid- $\beta$ and restores memory in an Alzheimer's disease mouse model. Sci. Transl. Med. 2015, 7, 278ra33, DOI: 10.1126/scitranslmed.aaa2512.

19. Burgess, A.; Dubey, S.; Yeung, S.; Hough, O.; Eterman, N.; Aubert, I.; Hynynen, K. Alzheimer disease in a mouse model: MR imaging-guided focused ultrasound targeted to the hippocam-pus opens the blood-brain barrier and improves pathologic abnormalities and behavior. Radiology. 2014, 273, 736-745, doi.org/10.1148/radiol.14140245.

20. Shi, L.; Palacio-Mancheno, P.; Badami, J.; Shin, D.; Zeng, M.; Cardoso, L.; Tu, R.; B. Fu. Quantification of transient increase of the blood-brain barrier permeability to macromolecules by optimized focused ultrasound combined with microbubbles. Int. J. Na-nomedicine. 2014, 9, 4437-4448, doi: 10.2147/IJN.S68882.

21. Kovacs, Z.; Kim, S.; Jikaria, N.; Qureshi, F.; Milo, B.; Lewis, B.; Bresler, M.; Burks, S.; Frank, J. Disrupting the bloodbrain barrier by focused ultrasound induces sterile inflammation. Proc Natl Acad Sci USA. 2017, 114(1), E75-E84, doi.org/10.1073/pnas.1614777114.

22. Abrahao, A.; Meng, Y.; Llinas, M.; Huang, Y.; Hamani, C.; Mainprize, T.; Aubert, I.; Heyn, C.; Black, S.E.; K. Hynynen. First-in-human trial of blood-brain barrier opening in amyotrophic lateral sclerosis using MR-guided focused ultrasound. Nat Commun. 2019, 10, 4373, doi.org/10.1038/s41467-019-12426-9.

23. Semyachkina-Glushkovskaya, O.; Esmat, A.; Bragin, D.; Bragina, O.; Shirokov, A.; Navolokin, N.; Yang, Y.; Abdurashitov, A.; Khorovodov, A.; Terskov, A.; Klimova, M.; Mamedova, A.; Fedosov, I.; Tuchin, V.; Kurths, J. Phenomenon of music-induced opening of the blood-brain barrier in healthy mice. Proc. R. Soc. B. 2020, 287, 20202337, doi.org/10.1098/rspb.2020.2337.

24. Semyachkina-Glushkovskaya, O.; Bragin, D.; Bragina, O; Yang, Y.; Abdurashitov, A.; Esmat, A.; Khorovodov, A.; Terskov, A.; Klimova, M.; Agranovich, I.; Blokhina, I.; Shirokov, A.; Navolokin, N.; Tuchin, V.; Kurths, J. Mechanisms of Sound-Induced Opening of the Blood-Brain Barrier. Adv Exp Med Biol. 2021, 1269,197-202, doi: 10.1007/978-3-030-482381_31.

25. Semyachkina-Glushkovskaya, O.; Postnov, D.; Penzel, T.; Kurths, J. Sleep as a Novel Biomarker and a Promising Thera-peutic Target for Cerebral Small Vessel Disease: A Review Focusing on Alzheimer's Disease and the Blood-Brain Barrier. Int. J. Mol. Sci. 2020, 21, 6293, doi.org/10.3390/ijms21176293.

26. Pavlov, A.N; Khorovodov, A.P; AMamedova, A.T.; Koronovski, A.A.; Pavlova, O.N.; SemyachkinaGlushkovskaya, O.V.; Kurths, J. Changes in blood-brain barrier permeability characterized from electroencephalograms with wavelets and fluctuation analysis. Eur. Phys. J. Plus. 2021, doi.org/10.1140/epjp/s13360-021-01593-8. 
27. Semenova, N.; Segreev, K.; Slepnev, A.; Runnova, A.; Zhuravlev, M.; Blokhina, I.; Dubrovsky, A.; SemyachkinaGlushkovskaya,O.; Kurths, J. Non-invasive analysis of blood-brain barrier permeability based on wavelet and machine learning approaches. Eur. Phys. J. Plus. 2021, 136:736, doi.org/10.1140/epjp/s13360-021-01715-2.

28. Pavlov, A.N.; Pavlova, O.N.; Semyachkina-Glushkovskaya, O.V.; J. Kurths J. Extended detrended fluctuation analysis: effects of nonstationarity and application to sleep data. Eur. Phys. J. Plus. 2021, 136:10, doi.org/10.1140/epjp/s13360020-00980-x.

29. Runnova, A.; Zhuravlev, M.; Ukolov, R.; Blokhina, I.; Dubrovski, A.; Lezhnev, N.; Sitnikova,E.; Kiselev, A.; Karavaev, A.; Selskii, A.; Semyachkina - Glushkovskaya, O.; Penzel, T.; Kurths J. Modified Wavelet Analysis of ECoGPattern as Promising Tool for Detection of the Blood-Brain Barrier Leakage. Scientific Reports. 2021 (accepted)

30. Kiviniemi, V.; Korhonen, V.; Kortelainen, J.; Rytky, S.; Keinänen, T.; Tuovinen, T.; Isokangas, M.; Sonkajärvi, E.; Siniluoto, T.; Nikkinen, J.; Alahuhta, S.; Tervonen, O.; Turpeenniemi-Hujanen,T.; Myllylä, T.; Kuittinen, O.; Voipio, J. Realtime monitoring of human blood-brain barrier disruption. PLoS ONE. 2017, 12, e0174072, doi.org/10.1371/journal.pone.0174072.

31. Pavlov, A. N.; Dubrovsky, A. I.; Koronovskii Jr, A. A.; Pavlova, O. N.; Semyachkina-Glushkovskaya, O. V.; Kurths, J. Extended detrended fluctuation analysis of sound-induced changes in brain electrical activity. Chaos Solitons Fractals. 2020, 139, 109989, doi.org/10.1016/j.chaos.2020.109989.

32. Pavlov, A. N.; Dubrovsky, A. I.; Koronovskii Jr, A. A.; Pavlova, O. N.; Semyachkina-Glushkovskaya, O. V.; Kurths, J. Extended detrended fluctuation analysis of electroencephalograms signals during sleep and the open-ing of the bloodbrain barrier. Chaos. 2020, 30, 073138, doi.org/10.1063/5.0011823.

33. Semyachkina - Glushkovskaya, O.; Chehonin, V.; Borisova, E.; Fedosov, I.; Namykin, A.; Abdurashitov, A.; Shirokov, A.; Khlebtsov, B.; Lyubun, Y.; Navolokin, N.; Ulanova, M.; Shushunova, N.; Khorovodov, A.; Agranovich, I.; Bodrova, A.; Sagatova, M.; Shareef, A.E.; Saranceva, E.; Iskra, T.; Dvoryatkina, M.; Zhinchenko, E.; Sindeeva, O.; Tuchin, V.; Kurths, J. Photodynamic opening of the blood-brain barrier and pathways of brain clearing pathways. J. Biophotonics. 2018, 11, e201700287, doi.org/10.1002/jbio.201700287.

34. Wang, H.; Lai T. Optimization of Evans blue quantitation in limited rat tissue samples. Sci. Rep. 2014, 4, 6588, doi:10.1038/srep06588.

35. Schindelin, J.; Arganda-Carreras, I.; Frise, E.; Kaynig, V.; Longair, M.; Pietzsch,, T.; Preibisch, S.; Rueden, C.; Saalfeld, S.; Schmid, B.; Tinevez, J.-Y.; Hartenstein, V.; Eliceiri, K.; Tomancak, P., Cardona A. Fiji: an open-source plat-form for biological-image analysis. Nature Methods. 2019, 9, 676-682, doi:10.1038/nmeth.2019.

36. Peng, C.-K. Buldyrev, S.V.; Havlin, S.; Simons, M.; Stanley, H.E.; Goldberger, A. L. Mosaic organization of DNA nucleo-tides. Phys. Rev. E. 1994, 49, 1685-1689.

37. Peng, C.-K.; Havlin, S.; Stanley, H.E.; Goldberger, A. L. Quantification of scaling exponents and crossover phenomena in nonstationary heartbeat time serie. Chaos. 1995, 5, 82-87.

38. Pavlov, A.N.; Abdurashitov, A.S.; Koronovskii Jr., A.A.; Pavlova, O.N.; Semyachkina-Glushkovskaya, O.V.; Kurths J. Detrended fluctuation analysis of cerebrovascular responses to abrupt changes in peripheral arterial pressure in rats. Com-mun. Nonlinear Sci. Numer. Simul. 2020, 85, 105232, doi.org/10.1016/j.cnsns.2020.105232.

39. Louveau, A.; Kipnis, J. Dissection and immunostaining of mouse whole-mount meninges. Protocol exchange. 2015, doi: 10.1038/protex.2015.047.

40. Chen, K.-B.; Kuo, E. Y.; Poon, K.-S.; Cheng, K.-S.; Chan, C.-S. Liu, Y.-C.; Lai, T.W. Increase in Evans blue dye extravasation into the brain in the late developmental stage. Neuroreport. 2012, 23, 699-701. doi:10.1097/WNR.0b013e3283556dcc.

41. Saria, A.; Lundberg J. M. Evans blue fluorescence: quantitative and morphological evaluation of vascular permeability in animal tissues. J Neurosci Methods. 1983, 8, 41-49. 
42. Saunders, N.; Dziegielewska, K.; Møllgård, K.; Habgood, M. Markers for blood-brain barrier integrity: how appropriate is Evans blue in the twenty-first century and what are the alternatives? Front. Neurosci. 2015, 9, 385, doi.org/10.3389/fnins.2015.00385

43. Ma, Q.; Ineichen, B. V.; Detmar, M.; Proulx, S. T. Outflow of cerebrospinal fluid is predominantly through lymphatic vessels and is reduced in aged mice. Nat Commun. 2017, 8, 1434, doi.org/10.1038/s41467-017-01484-6.

44. Semyachkina-Glushkovskaya, O.; Postnov, D.; Kurths, J. Blood-Brain Barrier, Lymphatic Clearance, and Recovery: Ariad-ne's Thread in Labyrinths of Hypotheses. Int. J. Mol. Sci. 2018, 19, 3818, doi.org/10.3390/ijms19123818.

45. Nedergaard, M. Garbage truck of the brain. Science. 2013, 340, 1529-1530, DOI: 10.1126/science.1240514.

46. Karran, E.; De Strooper, B. The amyloid cascade hypothesis: are we poised for success or failure? J. Neurochem. 2016, 139, 237-252, doi.org/10.1111/jnc.13632.

47. Doody, R.S.; Thomas,R.G.; Farlow,M.; Iwatsubo, T.; Bruno Vellas, B.; Joffe, S.; Kieburtz, K.; Raman, R.; Sun, X.; Aisen, P.S. Phase 3 trials of solanezumab for mild-to-moderate Alzheimer's disease. N. Engl. J. Med. 2014, 370, 311-321, DOI: 10.1056/NEJMoa1312889.

48. Salloway, S.; Sperling, R.; Fox, N. C.; Blennow, K.; Klunk, W.; Raskind, M.; Sabbagh, M.; Honig, L.S.; Porsteinsson, A.P.; Ferris, S.; Reichert, M.; Ketter, N.;Nejadnik, B.; Guenzler, V.; Miloslavsky, M.; Wang, D.; Lu, Y.; Lull, J.; Tudor, L. C.; Liu, E.; Ph.D., Grundman, M.; Eric Yuen,E.; Black,R.; M.D.Brashear, H. R. Two phase 3 trials of bapineuzumab in mild-to-moderate Alzheimer's disease. N. Engl. J. Med. 2014, 370, 322-333,DOI: 10.1056/NEJMoa1304839.

49. Sevigny, J.; Chiao, P.; Bussière, T.; Weinreb, P.H.; Williams, L.; Maier, M.; Dunstan, R.; Salloway, S.; Chen,T.; Ling, Y.; O'Gorman, J.; Qian,F.; Arastu,M.; Li,M.; Chollate, S.; Brennan, M. S.;Quintero-Monzon, O.; Scannevin, R.H.; Arnold, H.M ; Engber, T.; Rhodes, K.; Ferrero, J.; Hang, Y.; Mikulskis, A.; Grimm, J.; Hock, C.; Nitsch, R. M.; Sandrock A. The antibody aducanumab reduces A $\beta$ plaques in Alzheimer's disease. Nature. 2016, 537, 50-56, doi.org/10.1038/nature19323.

50. Xie, L.; Kang, H.; Xu, Q.; Chen, M.J.; Liao, Y.; Thiyagarajan, M.; O'Donnell, J.; Christensen, D.J.; Nicholson, C.; Iliff, J.J.; Takano, T.; Deane, R.; Nedergaard M. Sleep drives metabolite clearance from the adult brain. Science. 2013, 18, 342(6156),373-377, doi: 10.1126/science.1241224.

51. Ooms, S.; Overeem, S.; Besse, K.; Rikkert, M.O.; Verbeek, M.; Claassen, J.A. Effect of 1 night of total sleep deprivation on cerebro-spinal fluid $\beta$-amyloid 42 in healthy middle-aged men: a randomized clinical trial. JAMA Neurol. 2014, 71(8), 971-997,doi: 10.1001/jamaneurol.2014.1173. 